\title{
Natural product diversity of actinobacteria in the Atacama Desert
}

\author{
Mostafa E. Rateb $(\mathbb{D} \cdot$ Rainer Ebel $\cdot$ Marcel Jaspars
}

Received: 28 November 2017/ Accepted: 25 January 2018/Published online: 14 February 2018

(C) The Author(s) 2018. This article is an open access publication

\begin{abstract}
The Atacama Desert of northern Chile is considered one of the most arid and extreme environment on Earth. Its core region was described as featuring "Mars-like" soils that were at one point deemed too extreme for life to exist. However, recent investigations confirmed the presence of diverse culturable actinobacteria. In the current review, we discuss a total of 46 natural products isolated to date representing diverse chemical classes characterized from different actinobacteria isolated from various locations in the Atacama Desert. Their reported biological activities are also discussed.
\end{abstract}

Keywords Atacama desert - Actinobacteria . Natural products

\section{Introduction}

Natural products are considered a valuable resource for drug discovery due to their diverse chemical scaffolds which cannot be matched by any synthetic libraries. Screening of novel microorganisms from

M. E. Rateb ( $\square)$

School of Science \& Sport, University of the West of Scotland, Paisley PA1 2BE, UK

e-mail: mostafa.rateb@uws.ac.uk

R. Ebel · M. Jaspars

Marine Biodiscovery Centre, University of Aberdeen, Aberdeen AB24 3UE, UK neglected and underexplored habitats, particularly the extremobiosphere that includes desert biomes is one of the main strategies to discover new chemical scaffolds, and hence chemical diversity (Pidot et al. 2014; Horikoshi et al. 2011; Guo et al. 2015). This strategy was initiated by the discovery of thermorubin from a thermophilic actinomycete recovered from a soil sample in Italy in 1964. Thermorubin exhibited strong activity against Gram-positive bacteria, lower activity against Gram-negative bacteria and some activity against fungi (Craveri et al. 1964).

The Atacama Desert of northern Chile is the most arid and extreme environment on Earth, having evolved over 100 million years of aridity and around 15 million years of hyper-aridity (Gómez-Silva et al. 2008). Its core region was described as featuring "Mars-like" soils that were deemed too extreme for life to exist owing to extreme aridity, high levels of UV radiation, the presence of inorganic oxidants, areas of high salinity, and very low concentrations of organic carbon (Navarro-González et al. 2003). However, subsequent investigations made in the same hyper-arid core confirmed that several taxa of cultivable microorganisms, especially actinobacteria were recovered from this harsh environment (Crits-Christoph et al. 2013; Bull et al. 2016). Moreover, some of these actinobacteria were screened for their capability to produce new natural products. For decades, actinobacteria were considered to be one of the most prolific sources for the discovery of antibiotics, including the most important antimicrobial drug classes such as $\beta$-lactams, 
tetracyclines, rifamycins, aminoglycosides, macrolides, and glycopeptides that were successfully introduced into the market around 40 years ago and are still used today in clinical practice. Many antibiotic classes were discovered from different actinobacterial sources and several new ones are currently under clinical investigation (Genilloud 2017). Such antibiotics were discovered from marine and terrestrial actinobacteria through different bio-guided screening approaches using screens that targeted rare and novel actinobacteria, derived from the application of innovative isolation methods and genome-based approaches (Genilloud 2017).

In the current review, we will discuss the diverse chemical classes of secondary metabolites isolated and characterized from different actinobacteria isolated from a range of locations in the Atacama Desert. Additionally, we will highlight different biological activities associated with the identified chemical entities from these actinobacteria.

\section{Approaches for the discovery of secondary metabolites from the Atacama Desert}

Researchers have used different approaches for detecting new natural products from actinobacteria isolated from the Atacama Desert. The most common approach has been the bioassay-guided protocol based on screening the partition fractions of fermentation broths followed by size exclusion and HPLC purification of the bioactive compounds. Once obtained, the structure determination of these bioactive molecules has been conducted using NMR and other spectroscopic approaches.

Bioassay-guided isolation led to the discovery of new types of aminobenzoquinones, abenquines A, B1, $\mathrm{B} 2, \mathrm{C}$ and D (1-5) that featured a rare combination of benzoquinone and a range of amino acids (Schulz et al. 2011). Compounds 1-5 were obtained from Streptomyces sp. strain DB634 isolated from the soils of the Chilean highland of the Atacama Desert and exhibited weak antibacterial and antifungal activities and moderate selective inhibitory activity against type 4 phosphodiesterase (PDE4b) that could be a promising target for the investigation of inflammatory diseases (Schulz et al. 2011). A similar strategy led to the isolation of a new family of 22-membered macrolactones, atacamycins A-C (10-12) from Streptomyces leeuwenhoekii strain C38 isolated from a hyper-arid
Atacama Desert salt lake sediment (Nachtigall et al. 2011). Compounds 10-12 exhibited moderate inhibitory activities against type 4 phosphodiesterase (PDE4b2), and 10 showed moderate antiproliferative effects against adenocarcinoma and breast carcinoma cells (Nachtigall et al. 2011). Recently, bio-guided fractionation of the culture extract of Streptomyces asenjonii strain $\mathrm{KNN}$ 42.f led to the isolation of three new bioactive $\beta$-diketones, asenjonamides $\mathrm{A}-\mathrm{C}$ (33-35) which exhibited broad spectrum antibacterial effect against a panel of Gram-positive and Gram-negative bacterial strains (Abdelkader et al. 2018).

Chemical profiling using LC-HRMS and NMR screening and database dereplication is another approach that has been used for Atacama Desert strain prioritisation. Lentzea sp. strain $\mathrm{H} 45$ was isolated from a high-altitude Atacama Desert soil and shown to produce a specific pattern of secondary metabolites based on its LCMS and associated NMR profiles. Large scale fermentation of this strain led to the isolation of six new diene glycosides; lentzeosides A-F (26-31) (Wichner et al. 2017). This organism has been validly named as the type strain of Lentzea chajnantorensis (Idris et al. 2017).

In contrast to bioassay-guided and chemical profiling approaches, computational genome mining and more recently whole-genome sequencing are proving to be fast and inexpensive tools for strain prioritisation. Thousands of bacterial genome sequences together with a huge number of secondary metabolite gene clusters are currently available awaiting linkage to their encoded natural products. With the development of high-throughput sequencing methods and the vast amount of whole genome sequence data available, mining of genomes has become an important part to guide discovery and characterisation of novel chemistry. In this context, the type strain of S. leeuwenhoekii $\left(\mathrm{C} 34^{\mathrm{T}}\right)$ has been used as a model organism. Before its full taxonomic characterization and genome sequencing, an in silico genome mining strategy was used to discover four new ansamycin-type polyketides, chaxamycins A-D (6-9) produced by this strain. Aminohydroxybenzoic acid (AHBA) synthetase is a wellknown target in genetic screening for novel compounds including ansamycin antibiotics. Consequently, genome mining of S. leeuwenhoekii $\mathrm{C} 34$ targeting the gene encoding rifamycin-specific AHBA synthase led to the discovery of four new ansamycin-type polyketides 6-9 (Rateb et al. 2011a). Another class of compounds 
amenable to genome mining are ribosomally synthesized and post-translationally modified peptides (RiPPs). A recent example is the discovery of a lasso peptide through the identification of the lasso peptide cpt gene clusters, which was used in the successful isolation and identification of chaxapeptin from $S$. leeuwenhoekii strain C58 (Elsayed et al. 2015).

The OSMAC (one strain-many compounds) approach aims to maximize the productivity of a given microorganism through expression of silent or cryptic biosynthetic genes. The OSMAC strategy is considered a simple and cheap way to induce secondary metabolite production through varying the composition of the fermentation medium or varying fermentation parameters such as shaker speed, temperature, light, etc. The application of OSMAC to the strain S. leeuwenhoekii C34 successfully led to the isolation of three new metabolites, chaxalactins A-C belonging to the rare class of 22-membered macrolactone polyketides (Rateb et al. 2011b).

Co-cultivation is an experimental imitation of conditions in natural microbe communities at a laboratory scale which has repeatedly been used for enhancing the production of specific metabolites or the induction of new bioactive natural products that are not produced in axenic cultures (Dashti et al. 2014; Ebrahim et al. 2016). Co-culture screening was applied to the Atacama Desert-derived Streptomyces bullii $\mathrm{C}^{\mathrm{T}}$. The induction of biosynthetic pathways in Aspergillus fumigatus in response to co-cultivation with this strain led to the isolation of seven metabolites belonging to the diketopiperazine class of alkaloids, and two metabolites from the rare class of pseurotins. None of these compounds were observed in pure independent cultures of the fungus or the bacterium (Rateb et al. 2013). More recently, when A. fumigatus MR2012 and S. leeuwenhoekii $\mathrm{C} 34^{\mathrm{T}}$ were co-cultured, the bacterial strain suppressed the production of most of the fungal metabolites detected in the axenic culture. However, it induced the production of two fungal indole metabolites that were not traced before; the known terezine D and a new luteoride D featuring an oxazino[6,5- $b$ ] indole moiety that was not previously reported in nature. Additionally, the fungus suppressed all polyketide derivatives that were isolated from $S$. leeuwenhoekii $\mathrm{C} 34^{\mathrm{T}}$ but surprisingly, the bacterial metabolite chaxapeptin, which had not been previously detected in this strain, was produced at a significant level (Wakefield et al. 2017). In the microbial co- culture of A. fumigatus MR2012 and S. leeuwenhoekii C58, the bacterial strain completely suppressed the production of the fungal metabolites. However, the bacterium was induced to produce pentalenic acid and nocardamine which were not observed in the single bacterial culture. Additionally, the titre of the lasso peptide chaxapeptin (16), the sole secondary metabolite produced by $S$. leeuwenhoekii C58, was greatly increased (Wakefield et al. 2017).

\section{Chemical diversity of Atacama Desert-derived actinobacteria}

Aminoquinones

Abenquines A, B1, B2, C and D (1-5) are a group of aminoquinone-derived natural products that feature a rare combination of benzoquinone and a range of amino acids (Schulz et al. 2011). Compounds 1-5 were isolated from Streptomyces sp. strain DB634 which was recovered from a soil sample collected from the Salar de Tara in the Chilean highland of the Atacama Desert. The absolute configuration of amino acid moieties in 1-5 were assigned as L using the advanced Marfey's method. A literature search indicated that among known bacterial metabolites, the aminobenzoquinone substructure was found only in ansamycin-type antibiotics (Floss et al. 2011). Biosynthetically, the reaction of quinones with amino acids was reported to occur under mild experimental conditions without any enzymes involved (Morrison et al.1969). For this reason it is suspected that the $N$ acetyl-aminobenzoquinone might act as a Michael acceptor for the incorporation of amino acids in the structure of the abenquines. An alternative hypothesis suggests that laccase may be responsible for the biosynthesis of abenquines, since this enzyme was reported to mediate the adduct formation of amino acids to hydroquinones (Wellington et al. 2010). Compounds 1-5 exhibited a moderate and selective inhibitory effect against type 4 phosphodiesterase (PDE4b) with $\mathbf{1}$ and $\mathbf{5}$ being the most active, but about fourfold less active than the positive standard, rolipram. This could be regarded as a promising scaffold for anti-inflammatory drug discovery. Compounds 1-5 also demonstrated weak antibacterial activity but no antifungal activity against the tested strains (Schulz et al. 2011). 
Ansamycins

Ansamycins are a group of bacterial macrocyclic polyketides composed of a benzenic or naphthalenic chromophore bridged by an aliphatic ansa chain that is connected to the chromophore through an amide linkage (Prelog and Oppolzer 1973). Ansamycins are reported to exhibit strong antimicrobial activity against Grampositive and some Gram-negative bacteria. Some ansamycins show antiviral activity toward bacteriophages and poxviruses (Wrona et al. 2008). In addition, the ansamycin class of polyketides is known for its antitumor effects through the selective interaction with the ATP-binding pocket in the $N$-terminal domain of the heat shock protein 90 (Hsp90) (Porter et al. 2009).

Using genome-mining experiments that targeted the gene encoding 3-amino-5-hydroxybenzoic acid (AHBA) synthase, four ansamycin-type polyketides were isolated and characterised from $S$. leeuwenhoekii $\mathrm{C} 34^{\mathrm{T}}$ and named chaxamycins A-D (6-9) after the Laguna de Chaxa sampling site (Rateb et al. 2011a). The planar structure of chaxamycins was confirmed by advanced NMR and HRMS techniques. Owing to the difficulty of assigning the relative configuration of these types of compounds due to the conformational freedom of the ansa chain, the relative configuration of $\mathbf{6}$ was obtained by analysing its crystal structure using single-crystal X-ray diffraction. Using this information the relative configuration of the other three analogues was confirmed by comparing their ROESY spectra and coupling constants with that of $\mathbf{6}$ (Rateb et al. 2011a). The lack of a methyl group at the olefinic $\mathrm{C}-16$ next to the amide link is a characteristic structural feature of the new chaxamycins that distinguishes them from all previously known members of the naphthalenic ansamycin family. Compounds 6-9 exhibited strong antibacterial activities with 9 showing promising activity against a panel of clinical isolates of methicillinresistant Staphylococcus aureus. 6-9 also inhibited the intrinsic ATPase activity of human heat shock protein Hsp90 revealing promising antitumor activity with compound 6 about twofold less active than the positive standard, tanespimycin (17- $\mathrm{N}$-allylamino- 17 demethoxygeldanamycin). This latter result was corroborated by virtual docking studies which revealed that chaxamycins docked into the ATP binding pocket of Hsp90 where $\mathbf{6}$ exhibited the highest docking score (Rateb et al. 2011a).
Macrolactones

Macrolactones are a family of polyketides assembled by polyketide synthases and are characterised by 10-membered to 42-membered macrocycles which often contain one or more systems of conjugated double bonds. They have been reported from a wide range of marine and terrestrial organisms, and display a variety of biological activities. Smaller-sized macrolactones such as erythromycins (12-16 ring atoms) are named macrolides, and are widely used as anti-infective agents due to their potent inhibitory effect on bacterial protein biosynthesis. Larger-sized macrolactones exhibit a wide variety of biological activities including antifungal, insecticide, anthelmintic, antitumour, immunosuppressive or antiinflammatory action, while the 22-membered macrolactones mainly exhibit cytotoxic activity (Omura 2002).

As described above, bioactivity-guided screening of 21 Streptomyces strains isolated from a hyper-arid Atacama Desert lake sediment led to the isolation of a new family of 22-membered macrolactones named atacamycins A-C (10-12) from S. leeuwenhoekii strain C38 (Nachtigall et al. 2011). Compounds 10-12 exhibited moderate inhibitory activities against type 4 phosphodiesterase (PDE-4b2), while $\mathbf{1 0}$ also showed moderate antiproliferative effects against adenocarcinoma and breast carcinoma cells (Nachtigall et al. 2011). The application of OSMAC to the strain $S$. leeuwenhoekii $\mathrm{C} 34^{\mathrm{T}}$ led to the isolation chaxalactins A$\mathrm{C}(\mathbf{1 3}-\mathbf{1 5})$ belonging to the same class of 22-membered macrolactone polyketides (Rateb et al. 2011b). It is worth noting that strain $\mathrm{C} 34^{\mathrm{T}}$ was isolated from the same soil sample as strain $\mathrm{C} 38$ and that both belong to a well-defined 16S rRNA subclade, though the two strains differ slightly in their 16S rRNA gene sequences. The relative configuration of $\mathbf{1 3}$ was obtained by analysing its crystal structure using single-crystal X-ray diffraction, which in turn allowed for determining the relative configuration of the remaining analogues by comparing their ROESY spectra and coupling constants (Rateb et al. 2011b). Compounds 13-15 showed strong to moderate antibacterial effects against Gram-positive strains. The structures most closely related to atacamycins and chaxalactins are the 24-membered polyketides of the macrolactin family. To date, macrolactins A-V have been isolated from marine and soil bacterial isolates and have been shown to possess antibacterial, antilarval, antiviral, and antitumor activities (Gao et al. 2010). 
Peptides

Lasso peptides (LPs) are a rare class of ribosomally synthesized and post-translationally modified peptides (RiPPs) that feature a unique "lariat knot" structural motif (Zimmermann et al. 2014; Arnison et al. 2013). The post-translational modification includes an isopeptide bond forming a 7-9 amino acid $N$-terminal macrolactam ring through which the remaining $C$ terminal chain is threaded (Zimmermann et al. 2014). LPs are classified into three sub-classes based on their $N$-terminal residues and the number of disulfide bridges. Class I LPs have an $N$-terminal Cys and contain two disulfide bridges; class II LPs have an $\mathrm{N}$ terminal Gly and are devoid of disulphide bridges, and class III LPs have an $N$-terminal Gly and contain one disulfide bridge (Maksimov and Link 2014). Owing to their exceptional stability to enzymatic degradation and thermal or chemical denaturation, LPs are highly attractive as potential scaffolds for epitope grafting (Maksimov et al. 2012).

Using a genome mining approach, the interrogation of the genome of S. leeuwenhoekii strain C58 led to the detection of a gene cluster directing the biosynthesis of a new lasso peptide. Subsequent strain fermentation led to the isolation of chaxapeptin (16) which was fully characterized as a new lasso peptide by HRESIMS and NMR methods. Its absolute configuration was confirmed by the advanced Marfey's method. The class II lasso nature of $\mathbf{1 6}$ was confirmed by calculating its NOE restraint-based solution structure (Elsayed et al. 2015). The macrocyclic ring of $\mathbf{1 6}$ was very resistant to fragmentation under standard or vigorous MS conditions and to different enzymatic degradation under standard conditions. 16 exhibited inhibitory activity in cell invasion assays with A549 human lung cancer cells similar to that of sungsanpin, another class II lasso peptide with closely related structural features, produced by Streptomyces strain SNJ013 recovered from deep-sea sediment collected off the Korean coast (Um et al. 2013). As described above, the titre of $\mathbf{1 6}$ was greatly increased when $S$. leeuwenhoekii strain C58 was co-cultured with A. fumigatus MR2012. Additionally, the same fungus induced the production of $\mathbf{1 6}$ in S. leeuwenhoekii $\mathrm{C} 34^{\mathrm{T}}$ which was not traced before in this bacterial strain (Wakefield et al. 2017).

Diketopiperazines (DKPs) are cyclic dipeptides acquired by the condensation of two $\alpha$-amino acids.
They are abundant in nature being produced by fungi, bacteria, plants, and mammals, but many cases they may also be encountered as degradation products of polypeptides, especially during microbial fermentation processes. DKPs are characterised by a rigid backbone which can mimic a preferential peptide conformation. Therefore, they present small and conformationally constrained heterocyclic scaffolds which are stable to proteolysis and able to bind to a wide range of receptors, and thus are regarded as attractive scaffolds for drug discovery (Borthwick 2012). A series of DKPs was isolated from $S$. asenjonii strain $\mathrm{KNN}$ 42.f and identified as cyclo(LPro-L-Val) 17, cyclo(L-Pro-L-Phe) 18, cyclo(L-Pro-LTyr) 19, brevianamide F 20, cyclo(3-hydroxy-L-ProL-Leu) 21, cyclo(3-hydroxy-L-Pro-L-Phe) 22, and cyclo(3-hydroxy-L-Pro-L-Tyr) 23 (Abdelkader et al. 2018). Both 20 and 23 exhibited weak antibacterial effects against Gram-positive bacterial strains tested.

Microbial siderophores are a group of organic compounds, produced by different microorganisms under iron-limiting conditions to improve iron uptake from the surrounding environment and make it accessible to microorganisms. Siderophores also play a critical role in maintaining microbial life through the expression of virulence and development of biofilms under harsh or threatening conditions, and assist in the extraction of heavy metals from contaminated environments (Saha et al. 2016). Additionally, siderophores in many reported cases act as signalling molecules (auto-regulator/quorum sensing molecules) (Bertrand et al. 2014). Siderophores also have been reported to mediate selective delivery of antibiotics to antibiotic-resistant bacteria by a Trojan horse strategy (Huang et al. 2013). Interestingly, D'Onofrio et al. (2010) reported that modified synthetic media containing siderophores may be suitable for attempting to cultivate hitherto uncultured bacteria, which could lead to the discovery of novel previously non-accessible microorganisms. The major groups of siderophores include the catecholates (phenolates), carboxylates (e. g. derivatives of citric acid), and hydroxamates (Hider and Kong 2010).

Two representatives of the latter class, the known, deferrioxamine E (nocardamine, 24) and desferrioxamine B (25) were isolated from S. leeuwenhoekii $\mathrm{C} 34^{\mathrm{T}}$ when cultured under different fermentation conditions. 24 was obtained in large quantities reaching $100 \mathrm{mg} / \mathrm{L}$ 
in nutrient-deficient media (Rateb et al. 2011b). Recently, S. leeuwenhoekii strain C58 was induced to produce $\mathbf{2 4}$ when co-cultured with $A$. fumigatus
MR2012, while 24 was not observed in the axenic bacterial culture. This finding could indicate that $\mathbf{2 4}$ may have signalling effects (Wakefield et al. 2017).<smiles>CC(=O)NC1=CC(=O)C(NC(CC(C)C)C(=O)O)=CC1=O</smiles><smiles>CCC(C)[C@H](NC1=CC(=O)C(NC(C)=O)=CC1=O)C(=O)O</smiles>

3<smiles>CC(C)C(NC1=CC(=O)C=CC1=O)C(=O)O</smiles><smiles>CC(=O)NC1=CC(=O)C(NC(Cc2c[nH]c3ccccc23)C(=O)O)CC1=O</smiles>

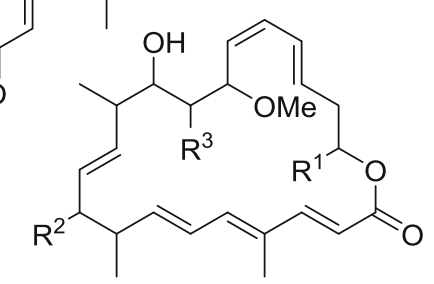<smiles>CCCCCC(C)=O</smiles><smiles>[R]c1c(C)c(O)c(C(=O)/C=C(\C)C([R])C)c2c1C(=O)C(I)=C(C)C2=O</smiles><smiles>C/C=C\C(=O)NCCC</smiles><smiles>C1CCOC1</smiles>

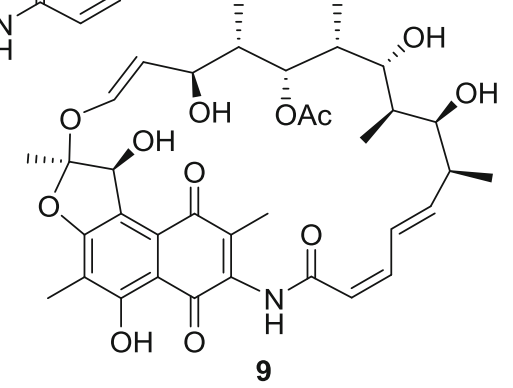<smiles>[R]</smiles>

$R^{2} \quad R^{3}$

10 (E)-but-1-ene $\mathrm{OMe} \mathrm{OH}$

11 (E)-but-1-ene $\mathrm{H} \quad \mathrm{OH}$

12 (E)-but-1-ene $\mathrm{H}$<smiles>[R]C=CC=CCC(C=CCC)OC(=O)C=CC(C)=CC=CC(C)CC=C</smiles>

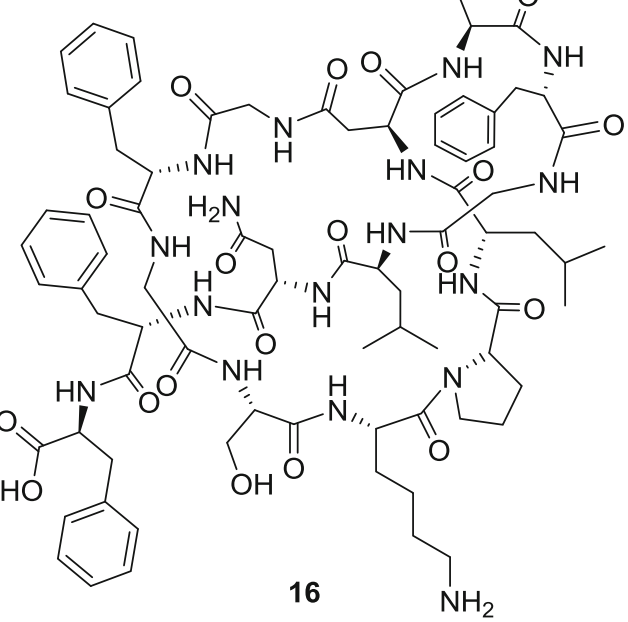

(E)-but-1-ene<smiles>[R]C1NC(=O)[C@@H]2CCCN2C1=O</smiles>

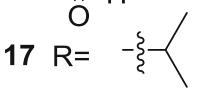

$18 \mathrm{R}=$ 埩<smiles>[R]Cc1ccc(O)cc1CSCC(=O)O</smiles><smiles></smiles>

$20 \mathrm{R}$

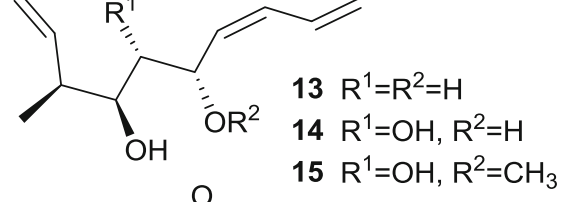<smiles>[R][Y9]Cc1ccc(O)cc1</smiles> 
<smiles>CC/C=C\C=C\CO[C@H]1O[C@H](C)[C@H](O)[C@H](O)[C@H]1O</smiles><smiles>CC/C=C\C=C\CO[C@@H]1O[C@H](C)[C@H](O)[C@H](O)[C@H]1O</smiles><smiles>CC/C=C\C=C\CO[C@H]1O[C@H](CO)[C@@H](O)[C@H](O)[C@H]1O</smiles><smiles>CC/C=C/C=C/CO[C@@H]1O[C@H](C)[C@H](O)[C@H](O)[C@H]1O</smiles>

29<smiles>C/C=C\CCO[C@H]1O[C@H](C)[C@H](O)[C@H](O)[C@H]1O</smiles>

30<smiles>CCCCCO[C@H]1O[C@H](C)[C@@H](O)[C@H](O)[C@H]1O</smiles>

31<smiles>CC/C=C\CCO[C@H]1O[C@H](CO)[C@@H](O)[C@H](O)[C@H]1O</smiles>

32<smiles>CCCC/C=C(\C)C(=O)C(C)C(=O)NCCO</smiles>

33<smiles>CCCC/C=C(\C)C(=O)[C@H](C)C(N)=O</smiles>

34<smiles>CCCCC1=C(C)C(=O)[C@](C)(N)C1=O</smiles><smiles>[R]O[R]([R])[R]#[R]</smiles>

$42 \mathrm{R}^{1}=\mathrm{H}, \mathrm{R}^{2}=\mathrm{OH}$<smiles>CC1(C)[C@H](O)[C@@H]2C=C(C(=O)O)[C@H]3CC[C@@H]1[C@H]32</smiles>

43<smiles>CC(=O)NCC(=O)c1c[nH]c2ccccc12</smiles>

44<smiles>OCCc1c[nH]c2ccccc12</smiles>

45<smiles>OCc1c[nH]c2ccccc12</smiles>

46
Diene glycosides

Chemical profiling by LC-HRMS and NMR screening and database dereplication allowed for the prioritisation of Lentzea sp. strain $\mathrm{H} 45$ which was isolated from a high-altitude Atacama Desert soil. The genus Lentzea was validly named over 20 years ago (Yassin et al. 1995), but despite this it has not featured in natural product chemistry studies. Large scale fermentation of this strain led to the isolation of six new diene glycosides, lentzeosides A-F (26-31) together with the known monoene glycoside Z-3hexenyl glucoside 32 (Wichner et al. 2017). The structures of the new metabolites were confirmed based on NMR and HRMS analyses, and their relative configuration was assigned based on ROESY correlations and coupling constant analyses. It is interesting to note that the lentzeoside class of diene metabolites was not reported previously from a microbial source, but only from plants (Miyase et al. 1988). When screened for their anti-HIV integrase activity at different concentrations, 28-30 displayed $\mathrm{IC}_{50}$ values of 21,16 and $21 \mu \mathrm{M}$, respectively while 26, 27, and 31 exhibited only weak activity. A standard HIV treatment regimen comprises a combination of different drugs that target different stages of the viral replication cycle to avoid the development of viral resistance due to its high mutation rate. In this context, the lentzeoside core could be a promising lead structure for the development of new antiviral drugs. 


\section{$\beta$-Diketones}

When screened against different bacterial isolates, only $S$. asenjonii strain KNN 42.f out of a collection of ten different Atacama Desert soil-derived actinobacteria showed strong antibacterial effects against Gram-positive and Gram-negative tested strains. Additionally, its extract showed specific UV and ${ }^{1} \mathrm{H}$ NMR patterns of secondary metabolites. Large scale fermentation of this strain followed by bio-guided fractionation resulted in the isolation of three new metabolites belonging to the rare $\beta$-diketone family of polyketides, asenjonamides $\mathrm{A}-\mathrm{C}(\mathbf{3 3}-\mathbf{3 5})$. The structures of the new compounds were elucidated based on HRESIMS and NMR analyses (Abdelkader et al. 2018). To the best of our knowledge, the $\beta$ diketone nucleus of $\mathbf{3 5}$ has not been reported in nature before. Compounds 33-35 exhibited significant antibacterial effects against Gram-positive strains with 35 showing comparable activity to that of tetracycline. Moreover, $\mathbf{3 5}$ exhibited strong activity against Gram-negative strains and moderate effects against Mycobacterium smegmatis (Abdelkader et al. 2018). These findings suggest 35 to be a good candidate for developing new broad-spectrum antibiotics.

\section{Aminoheptosyl glycosides}

During the bio-guided fractionation of the culture broth of $S$. asenjonii strain KNN 42.f, the structurally unique antitumor antibiotics spicamycins A-E (3640) were isolated in high purity for the first time (Abdelkader et al. 2018). This series of the bioactive acylated 4-aminoheptosyl- $\beta$ - $N$-glycosides features an adenine base, an unusual amino sugar, and aliphatic side chains ranging from 8 to $12 \mathrm{CH}_{2}$ groups ending in an isopropyl moiety. The structures of these compounds were fully characterised by extensive HRESIMS and NMR spectroscopic analyses. Spicamycins were initially characterized as a nonseparable mixture of seven compounds from the culture broth of Streptomyces alanosinicus strain 879-MT3 and reported as potent differentiation inducers of HL-60 human promyelocytic leukaemia cells at a concentration range of $250-600 \mathrm{ng} / \mathrm{mL}$ (Hayakawa et al. 1983). The structural moiety of these unique nucleoside antibiotics was confirmed through the total synthesis of one of the spicamycin congeners, SPM VIII (Suzuki et al. 2002). The sugar moiety in spicamycins was confirmed to be derived from the pentose phosphate pathway through isotopic enrichment studies that were conducted using a Streptomyces fimbriatus strain (Price et al. 2014). A series of synthetic spicamycin analogues was prepared and screened for their antitumor effect. Among them, KRN5500 (NSC650426) exhibited the highest activity and therapeutic index in human xenograft models of gastric, colon and oesophageal cancer with a response rate comparable to that of mitomycin $\mathrm{C}$, which prompted the start of phase I clinical trials (Gadgeel et al. 2003). Compounds 36-40 exhibited weak antibacterial effects against Gram-positive strains in the MIC range of $70-85 \mu \mathrm{g} / \mathrm{mL}$ but no effects against Gram-negative strains (Abdelkader et al. 2018).

Other natural product classes

Totomycin (also known as hygromycin A, 41), an aminoglycoside antibiotic first described from Streptomyces hygroscopicus in the 1950s and known for its inhibitory effect on bacterial ribosomal peptidyl transferase (Mann et al. 1953), and its congener 5"dihydrohygromycin A $\mathbf{4 2}$ were isolated and characterised from $S$. leeuwenhoekii strain $\mathrm{C} 34^{\mathrm{T}}$ when, in the course of applying the OSMAC approach, ISP3 medium was used for bacterial fermentation (Rateb et al. 2011b).

Pentalenic acid $\mathbf{4 3}$ is an angular triquinane sesquiterpene that was first isolated as a shunt pathway product of pentalenolactone biosynthesis (Seto et al. 1978), and later was obtained from many Streptomyces spp. as a co-metabolite of the pentalenolactone sesquiterpene family of natural products (Takamatsu et al. 2011). During co-culture of $A$. fumigatus MR2012 and S. leeuwenhoekii strain C58, the bacterium was induced to produce $\mathbf{4 3}$ which was not observed in the axenic bacterial culture under various fermentation conditions. LCMS analysis did not uncover any related sesquiterpene derivatives in the co-culture extract (Wakefield et al. 2017).

During bio-guided isolation of secondary metabolites from the antibacterial active fraction of $S$. asenjonii strain KNN 42.f, $N$-(2-(1H-indol-3-yl)-2oxoethyl)acetamide 44, 2-(1H-indol-3-yl)ethan-1-ol 45, and (1H-indol-3-yl)methanol 46 were isolated and identified using NMR and HRMS analyses. None 


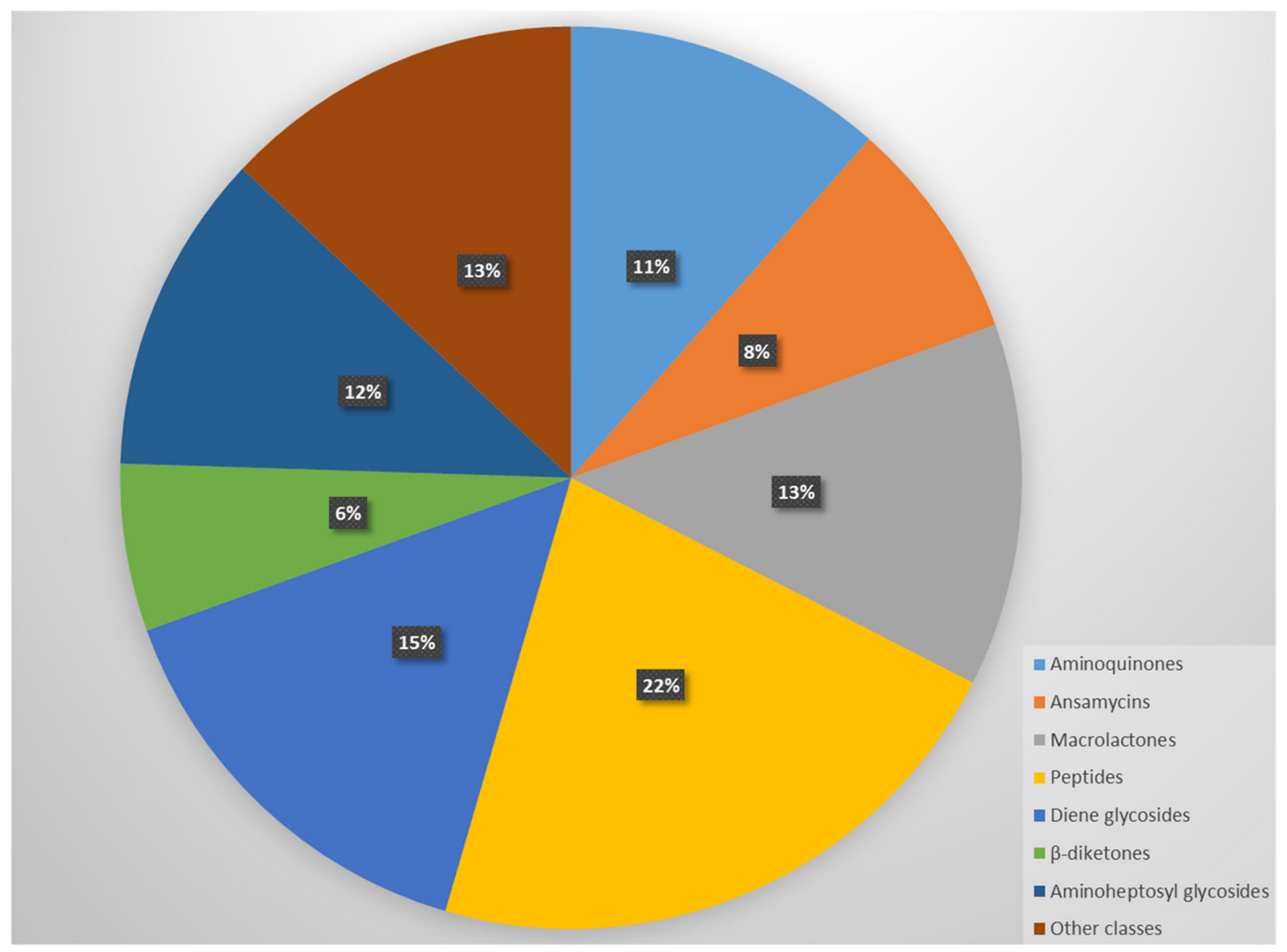

Fig. 1 Natural product diversity of actinobacteria isolated from the Atacama Desert soils

of these compounds exhibited antibacterial effects against a panel of bacterial strains (Abdelkader et al. 2018).

\section{Conclusion and highlights}

Although the Atacama Desert features the oldest, driest and most extreme hyper-arid desert soils that was previously considered too extreme for microbial life to exist, recent investigations have led to the discovery of taxonomically diverse cultivable actinobacteria from this harsh environment (Bull et al. 2016). To date, different approaches have been used for the detection of new natural products from these extremophilic and extremotolerant filamentous actinobacteria. Such efforts have led to the discovery of a total of 46 secondary metabolites representing diverse chemical classes, including alkaloids, peptides, polyketides, macrolides and terpenes. The major class of isolated metabolites belongs to peptides which represents about $22 \%$ of the isolated compounds (Fig. 1). The isolated metabolites have been shown to exhibit a range of biological activities including antibacterial, antiviral, and cytotoxic effects. These findings show that the Atacama Desert is an unexpectedly rich resource of actinobacterial diversity, and thus novel chemical diversity. This was clearly demonstrated by the isolation of such a high number of metabolites from only five actinobacterial strains. These metabolites showed a large range of novel structural features not previously reported before either from nature or from synthetic chemical approaches.

Open Access This article is distributed under the terms of the Creative Commons Attribution 4.0 International License (http://creativecommons.org/licenses/by/4.0/), which permits unrestricted use, distribution, and reproduction in any 
medium, provided you give appropriate credit to the original author(s) and the source, provide a link to the Creative Commons license, and indicate if changes were made.

\section{References}

Abdelkader MSA et al (2018) Asenjonamides A-C, antibacterial metabolites isolated from Streptomyces asenjonii strain $\mathrm{KNN}$ 42.f from an extreme-hyper arid Atacama Desert soil. J https://doi.org/10.1038/s41429-017-0012-0

Arnison PG et al (2013) Ribosomally synthesized and posttranslationally modified peptide natural products: overview and recommendations for a universal nomenclature. Nat Prod Rep 30:108-160

Bertrand S, Bohni N, Schnee S, Schumpp O, Gindro K, Wolfender J (2014) Metabolite induction via microorganism co-culture: a potential way to enhance chemical diversity for drug discovery. Biotechnol Adv 32:1180-1204

Borthwick AD (2012) 2,5-Diketopiperazines: synthesis, Reactions, Medicinal Chemistry, and Bioactive Natural Products. Chem Rev 112:3641-3716

Bull AT, Asenjo JA, Goodfellow M, Go'mez-Silva B (2016) The Atacama Desert: technical resources and the growing importance of novel microbial diversity. Ann Rev Microbiol 70:215-234

Craveri R, Coronelli C, Pagani H, Sensi P (1964) Thermorubin, a new antibiotic from a thermoactinomycete. Clin Med 71:511-521

Crits-Christoph A et al (2013) Colonization patterns of soil microbial communities in the Atacama Desert. Microbiome 1:28. https://doi.org/10.1186/2049-2618-1-28

D'Onofrio A, Crawford JM, Stewart EJ, Witt K, Gavrish E, Epstein S, Clardy J, Lewis K (2010) Siderophores from neighbouring organisms promote the growth of uncultured bacteria. Chem Biol 17:254-264

Dashti Y, Grkovic T, Abdelmohsen UR, Hentschel U, Quinn RJ (2014) Production of induced secondary metabolites by a co-culture of sponge-associated actinomycetes, Actinokineospora sp. EG49 and Nocardiopsis sp. RV163. Mar Drugs 12:3046-3059

Ebrahim W et al (2016) Metabolites from the fungal endophyte Aspergillus austroafricanus in axenic culture and in fungal —bacterial mixed cultures. J Nat Prod 79:914-922

Elsayed S et al (2015) Chaxapeptin, a lasso peptide from the extremotolerant Streptomyces leeuwenhokii strain C58 from the hyper-arid Atacama Desert. J Org Chem 80:10252-10260

Floss HG, Yu TW, Arakawa K (2011) The biosynthesis of 3-amino-5-hydroxybenzoic acid (AHBA), the precursor of $\mathrm{mC7N}$ units in ansamycin and mitomycin antibiotics: a review. J Antibiot 64:35-44

Gadgeel SM et al (2003) A phase I clinical trials of spocamycin derivative KRN5500 (NSC650426) using a phase I accelerated titration 2B design. Investig New Drugs 21:63-74

Gao CH, Tian XP, Qi SH, Luo XM, Wang P, Zhang S (2010) Antibacterial and antilarval compounds from marine gorgonian-associated bacterium Bacillus amyloliquefaciens SCSIO 00856. J Antibiot 63:191-193 and references therein

Genilloud O (2017) Actinomycetes: still a source of novel antibiotics. Nat Prod Rep 34:1203-1232

Gómez-Silva B, Rainey FA, Warren-Rhodes KA, McKay CP (2008) Atacama Desert soil microbiology. In: Dion P, Nautiyal CS (eds) Microbiology of extreme soils book series. Soil Biology. Springer, Berlin

Guo X, Liu N, Li X, Ding Y, Shang F, Gao Y, Ruan J, Huang Y (2015) Red soils harbor diverse culturable actinomycetes that are promising sources of novel secondary metabolites. Appl Environ Microbiol 81:3086-3103

Hayakawa Y et al (1983) Studies on the differentiation inducers of myeloid leukemic cells III. Spicamycin, a new inducer of differentiation of HL-60 human promyelocytic leukaemia cells. J Antibiot 36:934-937

Hider RC, Kong X (2010) Chemistry and biology of siderophores. Nat Prod Rep 27:637-657

Horikoshi K, Antranikian G, Bull AT, Robb FT, Stetter KO (eds) (2011) Extremophiles Handbook. Springer, Tokyo

Huang Y, Jiang Y, Wang H, Wang J, Shin MC, Byun Y, He H, Liang Y, Yang VC (2013) Curb challenges of the "Trojan Horse" approach: smart strategies in achieving effective yet safe cell-penetrating peptide-based drug delivery. Adv Drug Deliv Rev 65:1299-1315

Idris H, Imen N, Asenjo JA, Bull AT, Goodfellow M (2017) Lentzea chajnantorensis sp. nov., a very high altitude actinobacterium which produces novel dienes and was isolated from Cerro Chajnantor gravel soil in northern Chile. Antonie Van Leeuwenhoek 110:795-802. https://doi.org/10.1007/s10482-017-5

Maksimov MO, Link AJ (2014) Prospecting genomes for lasso peptides. J Ind Microbiol Biotechnol 41:333-344

Maksimov MO, Pan SJ, Link AJ (2012) Lasso peptides: structure, function, biosynthesis, and engineering. Nat Prod Rep 29:996-1006

Mann RL, Gale RM, Van Abeele RF (1953) Hygromycin II. Isolation and properties. Antibiot Chemother 3:12791282

Miyase T et al (1988) Studies on the glycosides of Epimedium grandiflorum MORR. var. thunbergianum (MIQ.). Chem Pharm Bull 36:2475-2484

Morrison M, Steele W, Danner DJ (1969) The reaction of benzoquinone with amines and proteins. Arch Biochem Biophys 134:515-523

Nachtigall J et al (2011) Atacamycins A-C, 22-membered antitumor macrolactones produced by Streptomyces sp. C38. J Antibiot 64:775-780

Navarro-González R et al (2003) Mars-Like soils in the Atacama Desert, Chile, and the dry limit of microbial life. Science 302:1018-1021

Omura S (2002) Macrolide antibiotics: chemistry, biology, and practice, 2nd edn. Academic Press, Boston

Pidot SJ, Coyne S, Kloss F, Hertweck C (2014) Antibiotics from neglected bacterial sources. Int $\mathrm{J}$ Med Microbiol 304:14-22

Porter JR, Ge J, Lee J, Normant E, West K (2009) Ansamycin inhibitors of Hsp90: nature's prototype for anti-chaperonetherapy. Curr Top Med Chem 9:1386-1418

Prelog V, Oppolzer W (1973) Ansamycins, new class of microbial metabolites. Helv Chim Acta 56:2279-2287 
Price NPJ, Furukawa T, Cheng F, Qi J, Chen W, Crich D (2014) Biosynthesis of 4-aminoheptose 2-epimers, core structural components of the septacidins and spicamycins. J Antibiot 67:405-414

Rateb ME et al (2011a) Chaxamycins A-D, bioactive ansamycins from a hyper-arid desert Streptomyces sp. J Nat Prod 74:1491-1499

Rateb ME et al (2011b) Diverse metabolic profiles of a Streptomyces strain isolated from a hyper-arid environment. J Nat Prod 74:1965-1971

Rateb ME et al (2013) Induction of diverse secondary metabolites in Aspergillus fumigatus by microbial coculture. RSC Adv 3:14444-14450

Saha M, Sarkar S, Sarkar B, Sharma BK, Bhattacharjee S, Tribedi P (2016) Microbial siderophores and their potential applications: a review. Environ Sci Pollut Res Int 22:3984-3999

Schulz D, Beese P, Ohlendorf B, Erhard A, Zinecker J et al (2011) Abenquines A-D: aminoquinone derivatives produced by Streptomyces sp. strain DB634. J Antibiot 64:763-768

Seto H, Sasaki T, Uzawa J, Takeuchi S, Yonehara H (1978) Studies on the biosynthesis of pentaleno-lactone II. Isolation of pentalenic acid and pentalenolactone $\mathrm{H}$. Tetrahedron Lett 19:4411-4412

Suzuki T, Suzuki ST, Yamada I, Koashi Y, Yamada K, Chida N (2002) Total synthesis of spicamycin. J Org Chem 67:2874-2880
Takamatsu S, Xu LH, Fushinobu S, Shoun H, Komatsu M, Cane DE, Ikeda H (2011) Pentalenic acid is a shunt metabolite in the biosynthesis of the pentalenolactone family of metabolites: hydroxylation of 1-deoxypentalenic acid mediated by CYP105D7 (SAV_7469) of Streptomyces avermitilis. J Antibiot 64:65-71

Um S, Kim YJ, Kwon H, Wen H, Kim SH, Kwon HC, Park S, Shin J, Oh DC (2013) Sungsanpin, a lasso peptide from a deep-sea streptomycete. J Nat Prod 76:873-879

Wakefield J, Hassan HM, Jaspars M, Ebel R, Rateb ME (2017) Dual induction of new microbial secondary metabolites by fungal bacterial co-cultivation. Front Microbiol 8:1284. https://doi.org/10.3389/fmicb.2017.01284

Wellington KW, Steenkamp P, Brady D (2010) Diamination by $\mathrm{N}$-coupling using a commercial laccase. Bioorg Med Chem 18:1406-1414

Wichner D et al (2017) Isolation and anti-HIV-1 integrase activity of lentzeosides A-F from extremotolerant Lentzea sp. H45, a strain isolated from a high-altitude Atacama Desert soil. J Antibiot 70:448-453

Wrona IE, Agouridas V, Panek JS (2008) Design and synthesis of ansamycin antibiotics. Comp Rend Chim 11:14831522

Yassin AF et al (1995) Lentzea gen. nov., a new genus of the order Actinomycetales. Int J Syst Bacteriol 45:357-363

Zimmermann M, Hegemann JD, Xie X, Marahiel MA (2014) Characterization of caulonodin lasso peptides revealed unprecedented $\mathrm{N}$-terminal residues and a precursor motif essential for peptide maturation. Chem Sci 5:4032-4043 